\title{
Multimodal ultrasonographic evaluation in a case with unossified primary synovial osteochondromatosis
}

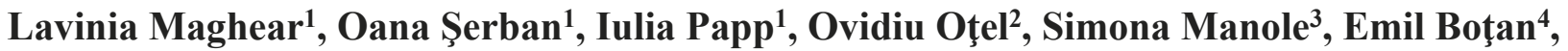 \\ Daniela Fodor ${ }^{1}$
}

${ }^{1} 2^{\text {nd }}$ Internal Medicine Department, "Iuliu Hatieganu" University of Medicine and Pharmacy, ${ }^{2}{ }^{\text {st }}$ Surgical Department, Emergency County Hospital, ${ }^{3}$ Radiology Department, "Iuliu Hatieganu" University of Medicine and Pharmacy, ${ }^{4}$ Pathology Department, Emergency County Hospital, Cluj-Napoca, Romania

\begin{abstract}
Primary synovial osteochondromatosis is a rare disorder and its diagnosis remains a challenge to the physician. We present the case of 36 year old patient with right knee monoarthritis in which ultrasound findings, corroborated with clinical and histopathological evidence confirmed the diagnosis of unossified primary synovial osteochondromatosis. The arthroscopy with synovectomy found multiple intra-articular loose bodies occupying the entire joint. The ultrasonographic findings were extensively evaluated.
\end{abstract}

Keywords: primary synovial osteochondromatosis; knee; ultrasonography

Primary synovial osteochondromatosis (PSO) (Reichel-Jones-Henderson syndrome) is a rare, benign, monoarticular disease, affecting the synovium. The etiology is uncertain; however, elevated levels of bone morphogenic protein, interleukin- 6 and vascular endothelial growth factor-A are found in the loose body lesions. The syndrome is characterised by multiple intra-articular cartilaginous loose bodies, uniform in size, that may grow, calcify or ossify. Cartilaginous bodies are a result of proliferation and synovial metaplasia; in severe cases they may occupy the entire joint space or infiltrate into adjacent structures. Patients usually are in the $4^{\text {th }}$ or $5^{\text {th }}$ decades of life and men to female ratio is $2: 1$ to $4: 1$. The knee is affected preferentially (up to $70 \%$ ); however, the disorder can arise in any other joint [1-6].

The most frequent clinical presentation includes pain, swelling and limitation of motion, often with slowly pro-

Received 10.03.2018 Accepted 22.05.2018

Med Ultrason

2018, Vol. 20, No 4, 527-530

Corresponding author: Daniela Fodor, $\mathrm{MD}, \mathrm{PhD}$

2nd Internal Medicine Department

"Iuliu Hatieganu" University of Medicine and

Pharmacy, Cluj-Napoca, Romania

2-4 Clinicilor str, 400006 ClujNapoca, Romania

E-mail: dfodor@umfcluj.ro gression (years) [3]. The ultrasonographic (US) features were not extensively evaluated. Recent studies describe hyperechoic foci, posterior acoustic shadowing, intraarticular fragments which may change in position during dynamic US examination. Power Doppler examination reveals avascular process [7]. We report the case of a young male with PSO, in which we used multiple US techniques in order to evaluate the specific features of this syndrome.

\section{Case report}

A 36 year old male patient, without previous medical history, non-smoker, was referred to our department for pain, swelling and limitation of motion at the right knee. The symptomatology started 3 years before presentation with diffuse, almost continuous, constrictive pain, intensified by effort, accompanied by progressive limitation of motion.

In this period the patient had few medical examinations and the most frequent etiologies of knee monoarthritis (septic arthritis, spondylarthritis, Lyme arthritis, crystal arthritis) were excluded. The magnetic resonance imaging (MRI) and US examinations found only unspecific findings (effusion and proliferated synovia). Local 


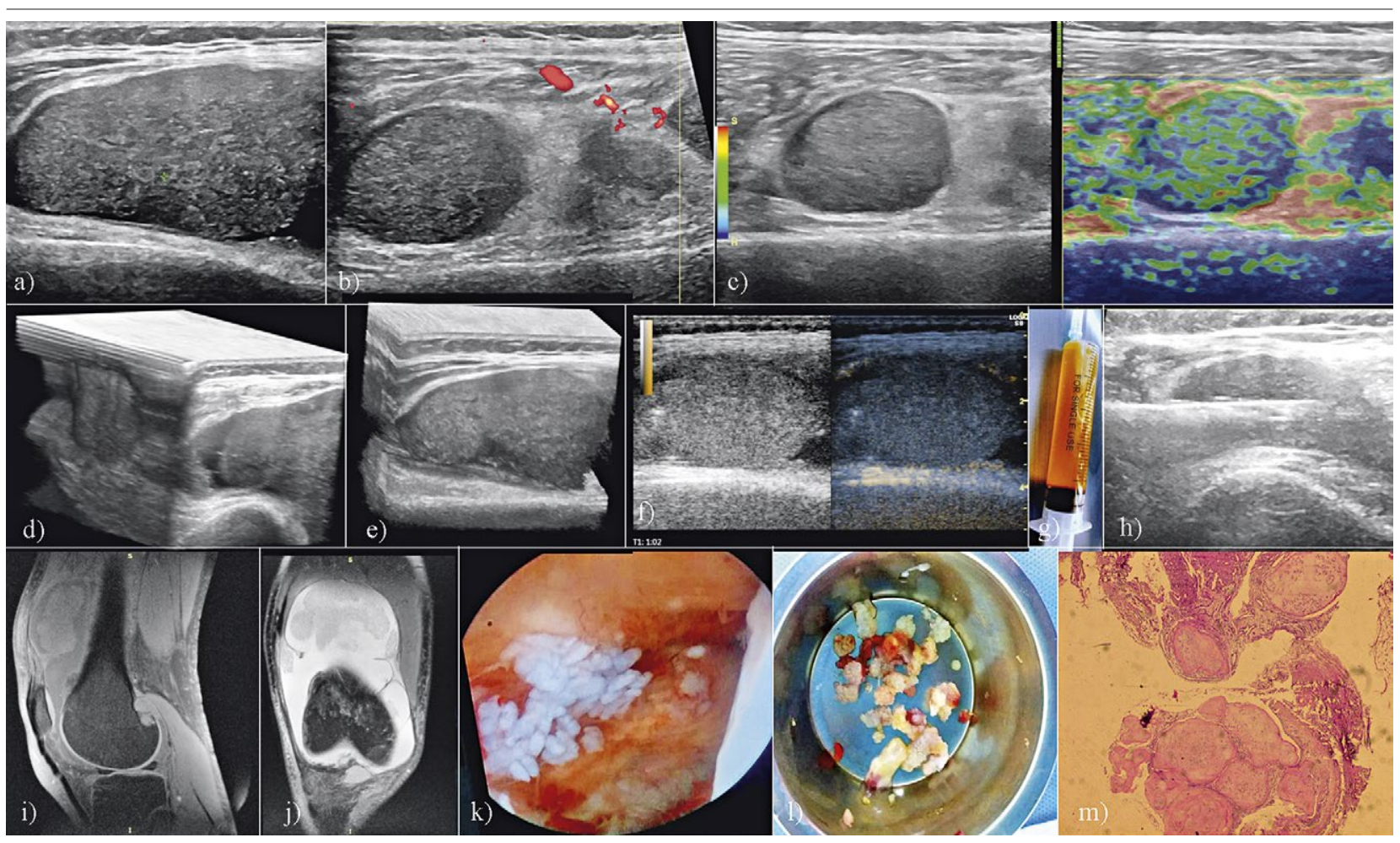

Fig 1. Grey-scale (a) and power Doppler ultrasonography (b) showed the presence of homogenous and non-vascularised masses in the suprapatellar bursa. The masses were stiff on strain elastography (c) and occupied the whole bursa on three dimensional examination ( $\mathrm{d}$ and e). The contrast-enhanced ultrasonography (f) confirmed that the masses were non-vascularized. The synovial fluid aspirated had a rust-colored aspect $(\mathrm{g})$ and an ultrasound-guided biopsy was performed (h). MRI examination confirmed the presence of large masses with intermediate signal intensity on T1-weighted images (i) and heterogeneously high signal intensity on T2-weighted images (j) with no enhancement

corticosteroid, analgesics and non-steroid anti-inflammatory drugs had no significant effect and the patient reported aggravated progressive limitation of motion. At admission a very painful, swollen knee with severe limitation of articular mobility was found but no other pathological signs. Biological exams, inclusive immunologic tests, showed no lab alterations. Knee conventional radiography was in normal range.

US examination was performed using GE Logiq S8 machine, with 6-15 MHz linear transducer. In the suprapatellar region of the right knee numerous echogenic and homogeneous masses surrounded by transonic effusion were identified (fig 1a). The masses had no vascularisation and at strain elastography a stiff appearance was found (fig 1b,c). Three-dimensional US revealed that the entire recess, including the parapatellar recesses, was filled by these masses (fig 1d,e) and contrast-enhanced US confirmed the absence of vascularisation (fig 1f). The aspirated fluid had a rustcolored aspect (high level of iron was biochemical detected in this fluid) (fig 1g). A US-guided biopsy was performed (fig 1h). A combination of MRI T1-weight- ed fast spin echo proton density (PD) imaging with and without frequency-selective fat suppression (fs) and MRI T2-weighted gradient echo sequences in the sagittal, axial, and coronal planes was realized. MRI confirmed the presence of the large masses (diameter up to $4.5 \mathrm{~cm}$ ), with intermediate signal intensity on T1-weighted images (fig 1i) and heterogeneously high signal intensity on T2-weighted images (fig $1 \mathrm{j}$ ) with no enhancement.

Arthroscopy was performed, with the removal of numerous loose bodies (fig $1 \mathrm{k}, \mathrm{l}$ ) and synovectomy. Histopathological the loose bodies were composed of hyaline cartilage lobules with slightly increased cellularity (fig $1 \mathrm{~m})$. The final diagnosis was of unossified PSO.

\section{Discussions}

PSO is an uncommon benign disorder in which hyaline cartilage nodules form in the synovial tissue of the joint, tendon sheath or bursa. These nodules are usually detaching from the synovium and can occupy the entire joint [1-3]. 
Our patient was a young male, with a long history disease and progressive aggravation of joint mobility. Previous studies showed that synovial chondromatosis can be highly aggressive and destructive, so a late diagnosis could severely affect the prognosis of the patient [2-5]. Microscopic appearance of PSO is composed of lobules of hyaline cartilage, surrounded by synovial lining (a two-cell layer of cuboidal epithelium). Sometimes, because of the metaplasia and pleomorphic cells, is very difficult to make the differential diagnosis between this benign pathology and chondrosarcoma [4].

Imaging findings depends on the stage of the disease and the presence of calcification or ossification of the cartilaginous bodies. For example, in $20-30 \%$ of the cases, wherein calcification is absent, there are no/non-specific radiographic findings (e.g. early osteoarthritis, erosions of adjacent structures, soft-tissue mass around the joint involved). Typical radiographic pattern includes multiple intraarticular chondral bodies with "ring-and-arc" chondroid mineralization and extrinsic erosion of bone. Our case had no calcification/ossification so there were no specific radiographic findings [5].

There are a few cases in literature in which the US findings in PSO are detailed. The presence of numerous small echogenic foci, representing the fronds of the synovium that have undergone metaplasia into cartilage were described $[6,7]$. We used all the US techniques (grey-scale, Doppler, elastography, three-dimensional US, elastography, contrast-enhanced US) in order to characterized this pathology. The homogeneity, stiff aspect and lack of vascularization were the main US findings in our case of un-ossified PSO.

MRI can be used for better evaluation of the extension of PSO within the joint and surrounding tissue. The pattern was characterized as lobulated, homogeneous, intermediate signal intensity similar to muscle appearence on T1-weighted images, with high signal intensity on T2weighted images. MRI can also evaluate marrow invasion, which is specific for malignant lesions, unusual for synovial chondromatosis [8].

Pigmented villonodular synovitis, synovial hemangioma and lipoma arborescens are conditions that can mimic synovial osteochondromatosis [9]. The necessity for a proper differential diagnosis and the impossibility of excluding a malignant process, were the reasons why we decided to perform a pre-surgical guided biopsy.

Management for PSO is mainly surgical and arthroscopic procedures are preferred. In our case, synovectomy was also realised, previous studies showing that this procedure gives better results compared to loose bodies removal alone [10].
Synovial chondromatosis can recur and when there is a recurrence it includes a fast growth and destruction of joints and a malignant degeneration must be considered $[11,12]$. It is well known that progression of synovial chondromatosis to chondrosarcoma is very rare. Distinction between these two conditions may be difficult using histology alone (the degree of cellularity and nuclear atypia may equal or exceed that seen in low grade chondrosarcoma), and should be based on clinical, imagistic and microscopic evidence [13].

In conclusion, US should be the first choice imaging technique in the evaluation of a clinically swollen or painful knee. Using the whole arsenal of US methods, the diagnosis and the plane for management of the local pathology can be generally accurately established. In unclear cases the need for other imaging techniques should be considered. Despite its lower frequency, in monoarticular pathologies, the PSO should be taken in consideration for differential diagnosis.

\section{References}

1. Karahan HG, Cetin O, Kurtulmuş A, Kayali C. Arthroscopic Management and Treatment of Synovial Chondromatosis and Talus Osteochondral Defect in the Ankle Joint. A Case Study. Ortop Traumatol Rehabil 2017;19:293-296.

2. Trevino M, Laks S, Kafchinski L, Sundarakumar DK, Smith CM. Intermetatarsal bursa primary synovial chondromatosis: case report and review of the literature. Skeletal Radiol 2017;46:1769-1773.

3. Boya H, Pinar H, Ozcan O. Synovial osteochondromatosis of the suprapatellar bursa with an imperforate suprapatellar plica. Arthroscopy 2002;18:E17.

4. ResnickD. Tumors and tumor-like lesions of soft tissues. In: Diagnosis of bone and joint disorders. 4th ed. Philadelphia, Pa: Saunders, 2002:4204-4273.

5. Murphey MD, Vidal JA, Fanburg-Smith JC, Gajewski DA. Imaging of synovial chondromatosis with radiologic-pathologic correlation. Radiographics 2007;27:1465-1488.

6. Harish S, Saifuddin A, Cannon SR, Flanagan AM. Synovial chondromatosis of the foot presenting with Lisfranc dislocation. Skeletal Radiol 2005;34:736-739.

7. Roberts D, Miller TT, Erlanger SM. Sonographic appearance of primary synovial chondromatosis of the knee. J Ultrasound Med 2004;23:707-709.

8. Kramer J, Recht M, Deely DM, et al. MR appearance of idiopathic synovial osteochondromatosis. J Comput Assist Tomogr 1993;17:772-776.

9. Paraschau S, Anastasopoulos H, Flegas P, Karanikolas A. Synovial chondromatosis: A case report of 9 patients. E E X O T 2008;59:165-169.

10. Yu GV, Zema RL, Johnson RW. Synovial osteochondromatosis. A case report and review of the literature. J Am Podiatr Med Assoc 2002;92:247-254. 
11. Ackerman D, Lett P, Galat DD Jr, Parvizi J, Stuart MJ. Results of total hip and total knee arthroplasties in patients with synovial chondromatosis. J Arthroplasty 2008;23:395400 .
12. Maini L, Sharma A. Synovial chondromatosis of knee. Indian J Med Res 2015;142:772-773.

13. Bertoni F, Unni KK, Beabout JW, Sim FH. Chondrosarcomas of the synovium. Cancer 1991;67:155-162. 